\title{
Creating Persona Skeletons from Imbalanced Datasets - A Case Study using U.S. Older Adults' Health Data
}

\author{
Haining Zhu \\ Pennsylvania State University \\ University Park, U.S.A. \\ hbz5037@psu.edu
}

\author{
Hongjian Wang \\ Twitter \\ San Francisco, U.S.A. \\ hongjianw@twitter.com
}

\author{
John M. Carroll \\ Pennsylvania State University \\ University Park, U.S.A. \\ jmc56@psu.edu
}

\begin{abstract}
Incorporating health personas for older adults into design processes can help designers accurately represent older adults by evoking empathy, facilitating consideration of health issues and needs, and reducing stereotype reliance. Toward this goal, we create a two-level quantitative methodology for constructing persona skeletons from imbalanced datasets. We demonstrate our methodology by constructing a set of 4 caremanagement personas for U.S. older adults via filtering and analyzing demographic, behavior risk factor, and chronic health conditions from 170,704 randomly sampled older adults in a national survey with imbalanced coverage (i.e. between unconditional \& conditional questions). We obtain 4 cluster centers for unconditional questions through K-means and iteratively dropping irrelevant features. Within each cluster, we analyze selected respondents for conditional questions. We synthesize results into persona narratives and provide a weighting scheme to quantitatively measure each persona's significance. We contribute a robust persona construction methodology, here applied towards representing older adults.
\end{abstract}

\section{Author Keywords}

BRFSS; personas; older adults; elderly; K-means clustering; rand index; silhouette score.

\section{CCS Concepts \\ -Human-centered computing $\rightarrow$ Interaction design; Inter- action design process and methods; User centered design; -Computing methodologies $\rightarrow$ Machine learning; Unsuper- vised learning; Cluster analysis;}

\section{INTRODUCTION}

User-centered design approaches are important when developing technologies for older adults [42, 51, 36], however incorporating older adults into user-centered design processes and research often requires special ethical and legal attention to protect their rights [26]. Researchers have argued that older adults are considered an at risk to being vulnerable "special class" $[49,32]$. More than $80 \%$ of US older adults have one

Permission to make digital or hard copies of all or part of this work for personal or classroom use is granted without fee provided that copies are not made or distributed for profit or commercial advantage and that copies bear this notice and the full citation on the first page. Copyrights for components of this work owned by others than ACM must be honored. Abstracting with credit is permitted. To copy otherwise, or republish, to post on servers or to redistribute to lists, requires prior specific permission and/or a fee. Request permissions from permissions@acm.org.

DIS '19, June 23-28, 2019, San Diego, CA, USA

(C) 2019 ACM. ISBN 978-1-4503-5850-7/19/06 . \$ \$15.00

DOI: https : //doi .org/10.1145/3322276. 3322285 or more chronic illnesses [53], and many older adults also experience physiological changes during aging (e.g., difficulty hearing or seeing can affect ability to give consent [49]) which may need to be considered to protect subjects' rights. Further exacerbating the issue of involving older adults in design research, ethics committees which rule on health and social issues may disadvantage qualitative research approaches [45].

In light of these special physiological, ethical, and legal considerations, utilizing elderly subjects in research may be difficult and undesirable if it burdens the subject. In such cases, proxies in the form of empirically grounded personas may stand-in for otherwise difficult to access subjects. Personas for older adults as proxies are a relatively underutilized design and development tool for consumer health technologies [31] and have faced general criticism in regard to representativeness (e.g., small sample sizes, predominantly qualitative studies, older adults being treated like a homogeneous group, etc.) [51].

The primary goal of this paper is to develop a cost-effective, quantitative method to construct health-representative personas. We use publicly available datasets because they reduce operation costs, limit designers' responsibilities in designing surveys and collecting responses (i.e., personas require constant updating to reflect current opinions and behaviors), and potentially reduce continued burdens upon participants in special classes. We aim to aid researchers and designers in exploring more deeply the issue of segmenting large, heterogeneous older adult populations through understanding the needs and behaviors of actual older adults. We use the world's largest continuously conducted health survey, the Behavioral Risk Factor Surveillance System (BRFSS [18]) 2016 to create health-representative personas for this widely heterogeneous group; it contains 275 health-related factors from 170,734 randomly sampled older adults in the USA and is updated annually, making it an ideal candidate for persona construction.

However, using publicly available datasets is challenging; 1) public surveys often contain unconditional questions of which all respondents are asked (i.e., demographics) and conditional questions which only apply to a portion of respondents (i.e., by previously asked questions, or questions selected at the discretion of a given State). This creates an imbalanced response rate between the two types of questions which makes quantitative analyses difficult. Large publicly available datasets like the NHANES ${ }^{1}$ [2], NHIS ${ }^{2}$ [3], and BRFSS [18], etc., share

\footnotetext{
${ }^{1}$ The National Health and Nutrition Examination Survey

${ }^{2}$ The National Health Interview Survey
} 
similar challenges. 2) Pre-existing data sources often contain extraneous data for the intended persona usage, so designers need a systematic way to select useful data for their projects. Towards our goal, we ask the following research questions:

- How can we address imbalanced data coverage in our dataset?

- How can we systematically select useful features to represent older adults?

We develop 4 personas from the 170,704 respondent sample set and then use human expertise on 30 samples from the same BRFSS surveys to verify the coherence of our persona clusters. Our paper expands upon recent HCI works involving methodologies for creating cost-effective personas. We offer the following contributions:

1. Our two-level method for constructing personas from preexisting public data sets with imbalanced data is the first to employ Silhouette value to determine cluster number and Rand Index to iteratively drop features (See Method 1 $\&$ 2). Our method also defines a quantitative measure for determining the significance of our different personas. This approach is suitable for other instances in which researchers are building personas from imbalanced data.

2. Using our method, we demonstrate how to construct a set of personas representing a varied range of attributes for US older adults derived from health-related risk behaviors and chronic health conditions; these may be applicable in their current forms or extended to specific contexts through supplemental data additions. ${ }^{3}$

\section{RELATED WORKS}

\section{Personas for US Older Adults}

Personas are hypothetical archetypal representations of actual target users with details such as names, demographic information, behaviors, goals, professions, etc., which are intended to represent a user and can be used in user-centered research $[13,23]$. Personas are often used with other complementary user-centered design methodologies [10], especially scenariobased design [46], as personas can provide a foundation on which a designer can build scenarios [23].

A significant body of HCI and design research has explored older adult persona use in other applications; researchers have used older adult personas to determine their web needs [12], evaluate usability of touch-phone design guidelines and recommendations [4], build user models for Ambient Intelligent technologies [9], improve quality of life and facilitate communication with caretakers [41], develop potential user interfaces [42], etc., but only a few persona studies to our knowledge have been focused primarily on older adults' health $[31,51]$.

Furthermore, many personas for older adults rely on data from outside of the United States of America (e.g., the European Union [9, 41, 42, 51], the People's Republic of China [31]), with only one notable example being generated from a large dataset for determining web behaviors within the USA [12]. Personas created based on data from outside of the USA, even

\footnotetext{
${ }^{3}$ The care-management personas, dataset and codebook, and python code are available at https://github.com/hainingzhu/Caremanagement-Persona.
}

if generalized, may not capture inherent differences caused by societal differences in healthcare provision (e.g., private care vs. socialized medicine) or general health factors (e.g., life expectancy trends [30]). Our work will utilize data from the USA to create "personas" which will better represent older adults in the USA so that researchers and designers can better extend empathy to these users.

\section{Personas Benefits and Criticisms}

Researchers have argued that using personas could increase empathy towards target users $[43,51]$; allow for complex user data to be present in all stages of the design process without the need for physical user presence; reduce designer bias and assumptions; and facilitate communication about user needs [43] and design choices [44]. In comparison to other user-centered techniques, personas are more engaging to designers and allow them to more strongly focus on target users, extrapolate from partial user knowledge to create more coherent narratives, and more explicitly determine their assumptions about users [23]. Furthermore, end users may not be available or appropriate for a given design process, and in such cases, personas can serve as an adequate, low-cost stand-in [33] which does not rely on stereotypes; however, stereotypes too may provide insights during design processes [15]. Finally, the use of personas benefits design teams by creating a common vocabulary regarding target users [48], and better facilitates the sharing and consensus of perceptions regarding users $[10,7]$. Misakiewicz et al. have a more detailed overview of the potential benefits of personas in user-centered design processes [36].

Persona use and construction methods have been controversial, which has caused researchers to devise new construction methods and a variety of personas (i.e., each defined in part by the methods used for construction [38, 43]. Notably, Cooper was criticized both for subjective methods (he relied on his experiences when constructing personas, which was not feasible for less-experienced researchers [23]), and subjective personas (as a result of not using empirical data $[44,35]$ ). Much of the attention on new persona methodologies has focused on better grounding persona construction and using empirical data. Even grounded personas may suffer from subjectivity in practice, however (e.g., from designer assumptions, experiences, etc. [5, 39]). Because of these flaws, personas may also lead designers into false feelings of understanding [20]. Some researchers further argue that even empirically grounded personas cannot be falsified, and thus could not be validated [11]. We intend to minimize subjectivity by utilizing a large quantity of survey data (BRFSS) to build empirically grounded persona skeletons and validate our results against a reserved testing set to show consistency.

\section{Current Methods of Creating Personas}

Many researchers have developed or improved construction methods for qualitative and quantitative personas [37, 16, 51]. Broadly speaking, contemporary construction methods include: user data collection, data segmentation and grouping, and writing persona descriptions and narratives [51]. Data collection can include both the utilization of pre-existing data [51] and the generation of task-specific data through surveys, interviews, or questionnaires [35, 24, 19]. Data is quantitatively and/or qualitatively analyzed (e.g., exploratory factor 
analysis [35] latent semantic analysis [37], affinity diagramming/utilizing grounded theory model [17], predictive models like J48 decision trees [6], data transcription with open coding and multidimensional scaling analysis [24], hierarchical clustering $[37,40]$ or partitional/k-means clustering $[34,51$, 19], weighted graphs [16], etc.). After persona attributes are identified via analysis, narratives are written $[37,17,27,38$, $19,14])$. Goodwin offers sound discussion of suitable means for building and presenting persona narratives [22]. During this stage, personas are given names, narrative descriptions of attributes, trait lists, a photo $[6,37,50]$, or multiple photos [27], and potentially foundation documents [23]. A persona narrative can be used as a form of validation for personas [17], but researchers might seek to further validate it (e.g., member checking [25] checking against interview data [35]). It is important to note that construction is flexible and that real-world applications may differ [10].

Summary: Like prior works $[19,34,51]$ on personas, our study applies K-means clustering to pre-existing data; unlike these prior works, we offer two methodological steps to address our specific challenges. We developed a two-level approach to construct personas while addressing imbalanced data coverages by employing Silhouette value and Rand Index to determine number of cluster centers and select important features to incorporate into persona skeletons.

\section{PRELIMINARY DATA EXTRACTION}

We utilize quantitative survey data from the BRFSS [18]; the system collects data from U.S. residents regarding their healthrelated risk behaviors, chronic health conditions, and use to preventative services. The BRFSS interviews over 400,000 adults from all 50 states through telephone interviews every year. In this paper, we focus only on older adults (i.e., 65 years or older) from the 2016 Survey, which accounts for 170,734 of the survey reports. The 275 questions in the BRFSS can be separated into the following 4 categories: 1) 86 unconditional questions about respondent general status (e.g., demographics, health-related quality of life, health care access), health conditions (e.g., cancer, stroke, diabetes), behavior risks (i.e., sleep, exercise, alcohol consumption, tobacco use), and use of preventative services (i.e., immunization, cancer screening, etc.); 2) 90 conditional questions similar to the unconditional questions which could be added, used, or modified at a state's discretion; 3) 26 questions related to landline or cell phone introductions, as well as record identification; and 4) 73 variables regarding calculated and weighting variables (e.g., respondent weight and height are asked in the demographic questions, but is then calculated as a BMI).

We endeavor to develop a comprehensive understanding of segmenting vast, heterogeneous older adult populations into health and behavior risk representing personas. To do this, we first removed extraneous data from our dataset. Omitted data includes: 1) 42 variables in the unconditional questions (e.g., health care access, oral health, immunization, seatbelt use, drinking and driving, breast and cervical cancer screening, prostate cancer screening, colorectal cancer screening, and HIV/AIDS); 2) 80 conditional questions (e.g., health care access, caregiver, menu labeling, marijuana use, influenza, HPV,

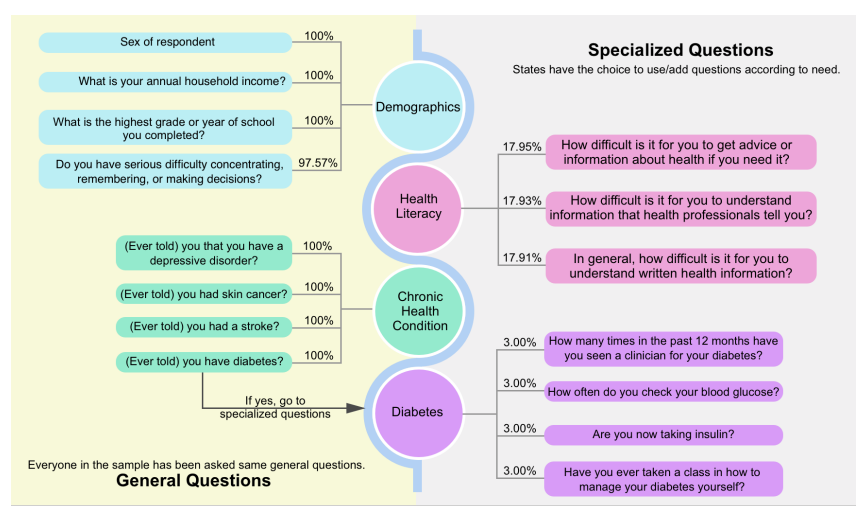

Figure 1: The BRFSS data from 2016 is divided into two types of question modules [1]. The left side represents unconditional questions, which includes 19 sets of questions asked of all respondents. The right side represents conditional questions which includes 25 sets of questions that could be added, used, or modified at a state's discretion. Response rates are denoted by the percentages beside the questions.

shingles, excess sun exposure, clinical breast exam, prostate cancer screening decision making, sexual orientation and gender identity, random child selection, childhood asthma prevalence, etc.); 3) all responses related to cell phone and landline introduction, as well as those regarding record identification; and 4) 69 weighting and calculated variables. However, 4 calculated variables (i.e., BMI, income, age, and education) were included as replacements to the weight, height, income, age, and education in the unconditional demographics module. Therefore, we have 44 unconditional questions, 4 calculated variables, and 10 conditional questions.

We observed a significant data coverage discrepancy between these two types of survey questions, which we refer to as Type 1 data (i.e., unconditional questions) and Type 2 data (i.e., conditional questions). We found that Type 2 data are usually scarce. For example, 36,313 of respondents reported that they have had diabetes (e.g., Type 1 data), which is equivalent to $21.3 \%$ of respondents. However, only $3 \%$ of the respondents with diabetes were selected by states to answer the conditional diabetes-related questions. To elaborate, Type 1 data samples are far more abundant than Type 2 data samples in our dataset as shown in Figure 1. With this observation in mind, we proposed a two-level method to construct personas. We provide a brief overview of our method at the end of this section. See Figure 1 for a more detailed example.

\section{Study Procedure Overview}

Building personas is essentially dividing users into different representative groups. This process is similar to the clustering problem in machine learning literature. A clustering problem takes a set of entities with their corresponding attributes and divides them into $k$ groups (i.e., clusters). Since "an appropriate dissimilarity measure is far more important in obtaining success with clustering than choice of clustering algorithm" [21], we utilize the K-means clustering method in this paper. The $\mathrm{K}$-means method is the most widely used clustering method and is suitable for clustering entities with a large number of attributes (i.e., features). 
To address the observed data discrepancy, we designed a twolevel procedure to create personas. We used different techniques to create two types of persona skeletons; this was motivated by the differences in the aforementioned two types of questions. Persona construction is difficult when a discrepancy exists between actual response rates, so a careful analysis of Type 2 data (i.e., conditioned statistical summarization of the state-dependent data) was necessary to resolve the discrepancy. We then combined the two-level persona skeletons to fully employ the data and build personas for older adults. The first persona skeleton depicts overall demographic and healthrelated risk behaviors, and is built on the complete dataset from the Type 1 data. Meanwhile, the second persona skeleton accounts only for specified health conditions, and is built from a smaller respondent pool from the reported Type 2 data.

With the challenges mentioned above, we developed a 6-step persona creation methodology. The methodology consists of the following steps: 1) use the k-means clustering method to build persona skeleton for Type 1 unconditional questions; 2) use the iterative feature selection method to systematically eliminate irrelevant features; 3 ) conduct statistical summarization on Type 2 conditional questions; 4) interpret K-means clusters to create personas; 5) measure the significance of identified personas; 6) employ human expertise to validate personas through reserved data samples.

\section{METHODS}

\section{K-Means Clustering of Unconditional Questions}

We use the K-means clustering method on the Type 1 data to construct the first level persona. From the clustering results we are able to construct an important component of the personas, called a persona skeleton. A detailed description of this procedure is given below.

Pre-process survey data. There are two motivations for data pre-processing. First, the survey data is noisy with a lot of missing values, such as "refused" and "don't know". We manually go through all survey questions and carefully decide how to map those missing values. For some cases, the missing value could be inferred with heuristics. For example, how many cigarettes have you smoked in the past 30 days. When the participants do not smoke, this question won't be asked and thus has a missing value. However, it is safe to fill such missing answers with 0 . For others that we could not determine adequately (e.g., "Now thinking about your physical health, which includes physical illness and injury, for how many days during the past 30 days was your physical health not good?" If the answer is 'Don't know/Not sure', 'Refused', 'Blank, Not asked or Missing'), we dropped these data points. In the end, we obtain 111740 samples out of 170704 .

Second, it is different to calculate distance across different types of questions. In our study, there are mainly three types of answers: binary, categorical, and continuous value. We convert them into the same scale, aka. binary values. For categorical questions, we create a binary feature for each category. For example, the answer to question BMI index could be underweight, normal, overweight and Obese. We create four features for this question as BMI_u, BMI_n, BMI_ow, and BMI_ob, each of which takes a binary value to represent the

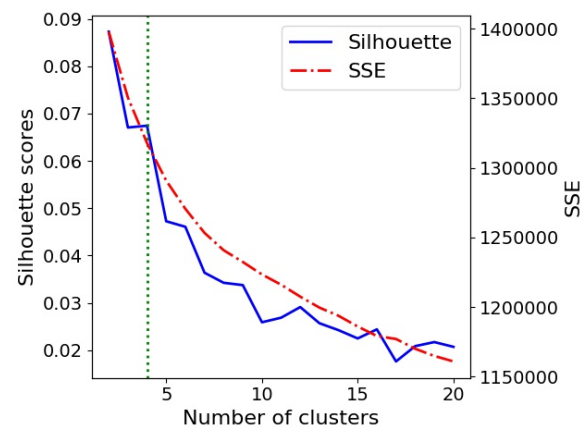

Figure 2: We use silhouette score and sum of squared error (SSE) to determine the optimal cluster numbers as 4 .

response categories. For continuous valued questions, we first use percentile to discretize the answers into different buckets. Then we further create a binary feature for each buckets, as in the categorical question.

Determine number of clusters. We use Euclidean distance function to run K-means on type 1 data. An important question is how many clusters should we have. We use silhouette score [47] and sum of squared error (SSE), as defined below, to determine the optimal cluster numbers.

Definition 1 (Silhouette). Assume the data has been clustered into $k$ clusters. For each data sample $i$, we use $a_{i}$ be the average distance between $i$ and all the other points in the same cluster. Let $b_{i}$ be the average distance between $i$ and all other points in any other clusters. The silhouette score for point $i$ is $s_{i}=\frac{b_{i}-a_{i}}{\max \left\{a_{i}, b_{i}\right\}}$.

The silhouette of a data sample is in the range of $[-1,1]$, where a larger value indicates better quality of clusters. We use the average silhouette of all data samples as silhouette score of the clustering results.

The sum of squared error (SSE) is the average distance of data sample to its corresponding cluster center. When cluster number is the same, the smaller SSE indicates better cluster quality. However, we could always reduce SSE by increasing the number of clusters. We plot silhouette score and SSE against number of clusters in Fig 2. We decide to use 4 clusters, for the following reasons. First, the silhouette score for 4 clusters is actually higher than that for 3 clusters, while the SSE is significantly reduced. Second, it is impractical to have more than 10 clusters.

\section{Iterative Feature Selection}

In order to efficiently build concise and accurate personas, we use a machine learning method to automatically select a subset of important questions. The reasons are 2 folds. First, the number of feature is huge. There are 48 unconditional questions and calculated variables, which are pre-processed into 125 features. Second, there is information redundancy among those questions.

Our method, called recursive feature elimination [29], drops the least important questions one by one from the original data. 
Table 1: Clustering centers of Type 1 data after feature selection.

\begin{tabular}{|c|c|c|c|c|c|c|c|c|c|c|c|c|c|c|c|c|c|c|c|}
\hline 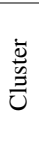 & 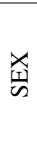 & 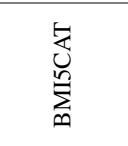 & 疍 & $\begin{array}{l}\sum_{0} \\
\text { Z } \\
Z\end{array}$ & 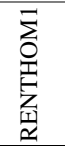 & 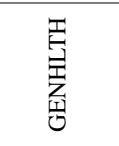 & 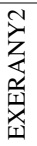 & 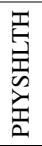 & 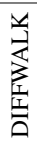 & 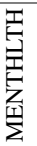 & 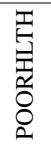 & 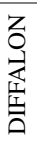 & 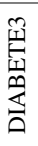 & 壱 & 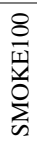 & 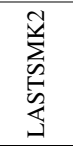 & 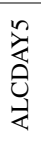 & 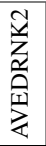 & 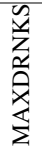 \\
\hline 1 & M & Overweight & Married & $50 \mathrm{k}+$ & Own & Very Good & $\mathrm{Y}$ & 0 & $\mathrm{~N}$ & 0 & 0 & $\mathrm{~N}$ & $\mathrm{~N}$ & 0 & $\mathrm{Y}$ & $10+$ & $8+$ & $1+$ & $2+$ \\
\hline 2 & $\mathrm{~F}$ & Obese & Widowed & $15 \mathrm{k}-25 \mathrm{k}$ & Rent & Fair & $\mathrm{N}$ & $6+$ & $\mathrm{Y}$ & $1+$ & $26+$ & $\mathrm{Y}$ & $\mathrm{Y}$ & $1+$ & $\mathrm{Y}$ & $10+$ & 0 & 0 & 0 \\
\hline 3 & $\mathrm{~F}$ & Normal & Married & $50 \mathrm{k}+$ & Own & Very Good & $\mathrm{Y}$ & 0 & $\mathrm{~N}$ & 0 & 0 & $\mathrm{~N}$ & $\mathrm{~N}$ & 0 & $\mathrm{~N}$ & Never & 8 & 1 & 1 \\
\hline 4 & $\mathrm{~F}$ & Overweight & Married & $50 \mathrm{k}+$ & Own & Good & $\mathrm{Y}$ & 0 & $\mathrm{~N}$ & 0 & 0 & $\mathrm{~N}$ & $\mathrm{~N}$ & 0 & $\mathrm{~N}$ & Never & 0 & 0 & 0 \\
\hline
\end{tabular}

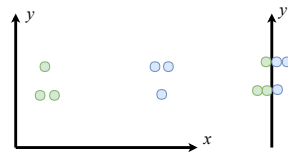

Figure 3: Example for feature selection procedure. Left: the original data and clustering results. Middle: clustering results after dropping feature $\mathrm{x}$. Right: clustering results after dropping feature $\mathrm{y}$.

We first use the K-means method to get the original partition of all users, denoted as $P_{\text {org }}$. Next, we drop one question from the existing question set and run another round of K-means to get a new partition $P^{\prime}$. A rand index measure $r i$, defined in Definition 2, is calculated to quantify the difference between $P$ and $P^{\prime}$. We aim to find a question to remove, after which the clustering results change as little as possible. We repeat the process above for $\mathrm{k}$ rounds to drop $\mathrm{k}$ questions in total.

DEFINITION 2 (RAND INDEX [28]). Rand index is the number of agreements between two partitions over the total number of pairs. For the same set of data samples, we have two clustering results $P$ and $P^{\prime}$. We define the following quantities: $a$ is the number of pairs that are in the same cluster in both $P$ and $P^{\prime} ; b$ is the number of pairs that are in different clusters in both $P$ and $P^{\prime} ; c$ is the number of pairs that are in the same cluster in $P$, but in different clusters in $P^{\prime} ; d$ is the number of pairs that are in different clusters in $P$, but in the same cluster in $P^{\prime}$. Rand index is $r i=\frac{a+b}{a+b+c+d}$.

We give an example in Figure 3 to intuitively explain the feature selection motivation and the semantics behind rand index. Rand index is in the range of $[0,1]$. A high rand index means that two clustering results are similar. In this example, the original data have two features $\mathrm{x}$ and $\mathrm{y}$, and they are clearly clustered into green and blue two clusters. First, we try to drop feature $\mathrm{x}$, and the clustering results are on the right, which is clearly different from the original clustering results. Rand index for dropping feature $\mathrm{x}$ is $\frac{7}{15}$. Next, if we try to drop feature $y$, we see that the clustering result, which is in the middle, has a rand index of 1 . Clearly, the feature y contributes less information in the original clustering. According to our method, we will choose to drop feature $y$.

In our study, we empirically dropped the features which have a corresponding rand index greater than 0.9. These values indicated that most participants provided the same or similar answers toward a question. In other words, we dropped the least important questions for contributing to differentiation between clusters. Using this method, we dropped 29 questions. In the end, we have 19 questions shown in Table 1.

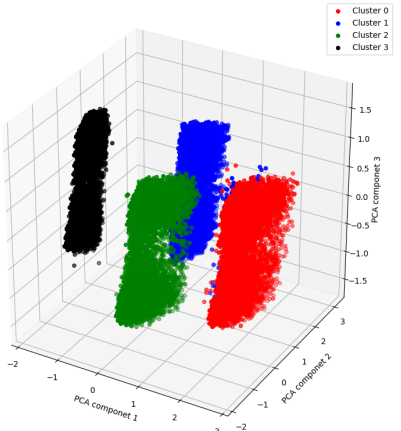

Figure 4: 3D visualization of Type I K-means results. We apply PCA and only take the top 3 components.

Visualize clustering results as sanity check. Although unnecessary for our persona skeleton construction method, we use Principal Component Analysis (PCA) and plot the results in Figure 4 to intuitively present readers our clustering results. Since the remaining clustering data still have 66 features, which will result in a 66-dimension visualization, we here employ a widely used dimension reduction method - PCA [52] only for the purpose of a sanity check. We take the top three components of the PCA results, which preserves the most information in original data, while enabling 3D visualization. Notice that PCA transforms the original data into new vector space, therefore it is difficult to interpret the semantics of PCA components. Further note that we did not interpret the PCA or variances because PCA was not used in our construction; this visualization is only for the purposes of our own sanity check and aiding readers' comprehensions. From Fig 5, we should observe that the data are well separated into 4 clusters, which indicates the good quality of our clustering analysis results.

Construct the persona skeleton. The personas are constructed from the centers of each cluster. The K-means clustering groups all respondents into 4 groups. Take cluster 1 as an example, which includes 19512 respondents. We calculate the average of 19512 answers to derive the center of this cluster, and this center is used as the representation of cluster 1.

\section{Summarization of Conditional Questions}

Type 2 conditional questions provide more details regarding peoples' health beliefs and comprehension, thus enhancing our personas skeleton. We have selected 3 groups of questions that fit our interests and goals. These three question groups are: Cognitive Decline, Emotional Support and Life Satisfaction, and Health Literacy. We conduct a statistical summarization on type 2 conditional questions for each type 1 cluster. Within each Type 1 cluster, we find the subset of participants that are selected for Type 2 questions. We then identify the mean value 
of each Type 2 question. The details of summarization over all respondents to each conditional question group are shown in Table 2.

\section{Persona Narrative Construction}

Using our persona skeletons and human expertise, we build our care management personas with the following 5 components: 1) fictitious names and pictures, 2) demographics, 3) characteristics labels, 4) background narratives, and 5) health related quality of life scales. Personas shown in Figure 5.

We flesh out our personas using popular names from the 40s' and pictures of representative older adults to make them more human-like. We interpret cluster centers (see Table 1 and 2) to construct our personas' demographics, characteristics labels, backgrounds narratives, and quality of life scales. For example, locations within the USA for personas are chosen at random by a researcher because locations are considered insignificant by our methods, and thus dropped. With the amount of data we use, noise and isolated differences are reduced significantly, but users of our methods may find it useful to create more targeted persona skeletons by focusing specifically on smaller populations (e.g., older adults just in the North East). Below, we provide a more detailed account of our skeleton interpretation process.

We first interpret the mean value of each question according to the BRFSS 2016 Codebook [18]. For example 'GENHLTH' refers to the question, "Would you say that in general your health is..." Underneath the question the value rubric lists that: '1' means 'Excellent', '2' means 'Very Good', '3' means 'Good', '4' means 'Fair', and '5' means 'Poor', respectively. Note that '7' and '9' stand for "don't know" or "refuse to answer", which are already dropped from our data. We examine the cluster centers for all 5 responses, and credit the ones with the highest value. For example in cluster 2, the mean values are $0.016,0.072,0.259,0.413$, and 0.240 , respectively. In this cluster, the majority responded with ' 4 ,' and ' 3 ' and ' 5 ' responses are somewhat equally distributed. We thus interpret cluster 2's general health as '4,' which is 'fair'.

There is one notable exception to this procedure, when all clusters have the same value for a given question. One example is 'RENTHOM1' (i.e., "Do you own or rent your home?"), where ' 1 ' refers to 'Own,' '2' refers to 'Rent,' and ' 3 ' refers to 'Other Arrangement' in the codebook. All of our clusters' have the highest mean value in the ' 1 ' category (i.e., in the 'Own' category; cluster 1 is 0.906 , cluster 2 is 0.716 , cluster 3 is 0.904 , cluster 4 is 0.872 ). The reason is that underlying data distribution are skewed toward category 1 . In this case, we use the average value $\frac{0.906+0.716+0.904+0.872}{4}=0.849$ as cutoff threshold, and thus round 0.716 in cluster 2 down to 0 . We follow the same procedure to interpret all the remaining questions. We create the scale regarding health status, chronic conditions, etc., on the bottom right, which represents our personas' health status.

\section{Determining the Significance of Each Persona}

After obtaining the personas for older adults, we further analyze the importance of different personas. The reason for this is that different personas should carry different levels of
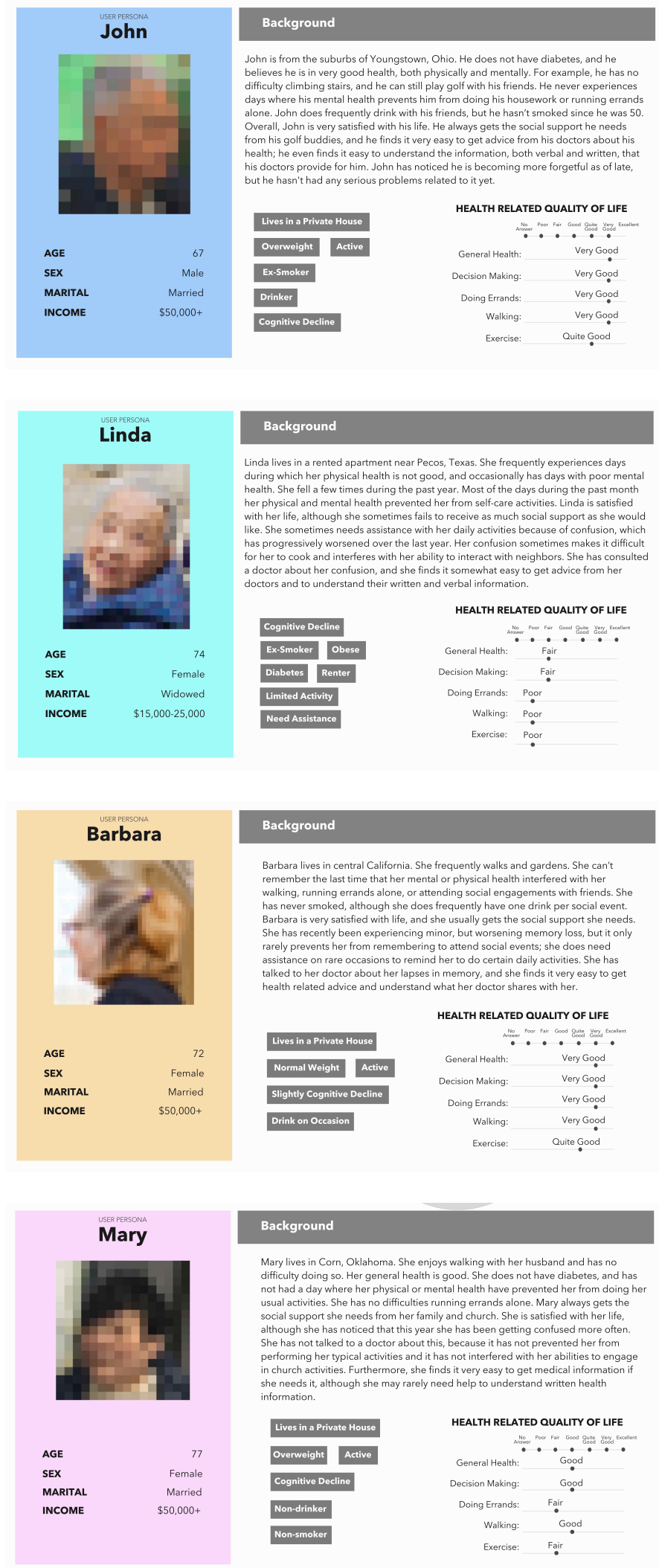

Figure 5: Care-management personas for U.S. older adults (John represents cluster 1, Mary representing cluster 4). 
Table 2: Mean values of respondents that have been selected to answer the given questions. Count shows how many respondents are in the given clusters.

\begin{tabular}{|c|c|c|c|c|c|c|c|c|c|c|c|}
\hline 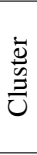 & 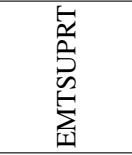 & 壳 & 量 & $\begin{array}{l}\text { Z } \\
\text { 点 } \\
\text { 合 } \\
\text { 点 }\end{array}$ & 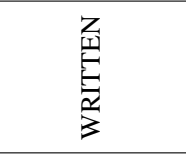 & 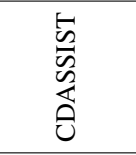 & 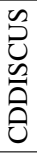 & 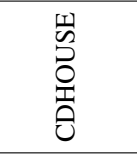 & 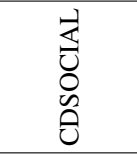 & $\sum_{\substack{\infty \\
0}}^{\infty}$ & 芯 \\
\hline 1 & Always & Very satisfied & Very easy & Very easy & Very easy & Never & $\mathrm{N}$ & Never & Never & $\mathrm{Y}$ & 5188 \\
\hline 2 & Sometimes & Satisfied & Somewhat easy & Somewhat easy & Somewhat easy & Sometimes & $\mathrm{Y}$ & Sometimes & Sometimes & $\mathrm{Y}$ & 5883 \\
\hline 3 & Usually & Very satisfied & Very easy & Very easy & Very easy & Rarely & $\mathrm{Y}$ & Never & Rarely & $\mathrm{Y}$ & 7609 \\
\hline 4 & Always & Satisfied & Very easy & Very easy & Somewhat easy & Never & $\mathrm{N}$ & Never & Never & $\mathrm{Y}$ & 10617 \\
\hline
\end{tabular}

significance in later design stages. A quantitative importance measure is able to prioritize different needs from different personas. Here we use the proportion of participants who fall into each persona as a measure of the significance, as shown in Table 3. The intuition is that the more people fall into a persona group, the more importance said group should have.

\section{Validation}

We use a validation set to verify that our personas are reasonable. From the dataset, 30 respondents are randomly sampled and reserved as the validation set. The 111,740 training set respondents' data are used to build the personas. We utilize human expertise (i.e., 2 authors and 2 invited researchers) to assign the 30 testing respondents to corresponding persona groups. Our initial agreement for classifying the 30 reserved data entries was $69.2 \%$; after discussion, the agreement rate increased to $92.5 \%$. This procedure can validate that our personas are reasonable, because the significance of personas determined by clustering method reasonably matches with domain knowledge of human expertise.

We provide the following three examples to show the testing set's correspondence to our personas: 1) Test subject 79911 is a 69-year-old female who is widowed, and has an income between $15 \mathrm{k}-25 \mathrm{k}$, believes she has fair general health, and smokes but does not drink. We matched her with persona 2 (Linda). 2) Test subject 227818 is a 75 -year-old female who is overweight, does not have diabetes, believes she has very good general health, and smokes and drinks. We matched her with persona 1 (John); 3) Test subject 276267 is a 69-yearold non-smoking and non-drinking male. He does not have physical or mental issues, and believes he has good general health; we matched him with persona 4 (Mary). The overall statistical weights (i.e., as determined by groupings based on the 4 humans' expertise) of the 30 respondents are shown in Table 3. The data demonstrate that human determined percentages match reasonably well with the quantitatively determined weights in Table 3. Such correspondence and consistency

Table 3: Validate the significance of identified persona. We hold out 30 respondents as validation set, and ask human experts to map these 30 respondents into corresponding persona groups. The raw counts and percentages of respondents for different persona groups are shown below.

\begin{tabular}{|c|r|r|r|r|}
\hline & \multicolumn{2}{|c|}{ Training } & \multicolumn{2}{c|}{ Validation } \\
\hline cluster & \#Resp & Weight & \#Resp & Weight \\
\hline 1 & 19512 & $17.46 \%$ & 6 & $20 \%$ \\
\hline 2 & 20352 & $18.21 \%$ & 6 & $20 \%$ \\
\hline 3 & 30122 & $26.96 \%$ & 7 & $23 \%$ \\
\hline 4 & 41754 & $37.37 \%$ & 11 & $37 \%$ \\
\hline
\end{tabular}

offers validation for our persona clusters and weights, thus indicating that our construction methods are reasonable.

\section{DISCUSSION}

In accordance with the prior works of researchers who construct personas for older adults through analysis of pre-existing survey data [51], our work demonstrates the efficacy of our two-level method for producing care-management personas for older adults in the USA. However, analyzing repositories of public data often comes with inherent challenges, such as data discrepancies and extraneous data. Data discrepancies arise in part from the design of the BRFSS survey, and the BRFSS covers features go beyond our needs, which make purposeful analyses, at least by humans, difficult and tedious. As seen in this paper, our approach was effective in constructing care-management personas despite these challenges, and we believe our two-level approach and iterative feature selection could tackle similar challenges in other datasets and applications (i.e., NHANES[2], NHIS[3], etc.). Despite these challenges, we see an opportunity in the fact that the BRFSS will continue to be updated on a yearly basis. Personas must be constantly updated to ensure that they maintain representativeness to the target demographic as the demographic changes, and as the BRFSS is a yearly survey with a significant number of respondents, we will have ample opportunities to update our care-management personas to ensure representativeness as well as to allow their further validation.

Corroborating Persona Validation. Although we discussed our cross-validation in a prior section (i.e., and note high initial and post-discussion inter-rater cross validation and weight consistencies), we note that our personas have actually undergone two forms of validation. As other researchers have noted, the ability to write a persona narrative is a form of validation for the persona [16]; notably, if meaningful narratives can be written without conflicting data, the persona is more likely valid than if meaningful narratives cannot be written. We experienced no difficulty in writing our persona narratives, and therefore note this "validation" as well as our favorable crossvalidation are corroborating evidence to support the validity of our personas and the theoretical soundness of our method.

Using Public Datasets. Questions may arise regarding whether data from the BRFSS, which was not intended for care-management persona construction, would create accurate portrayals or proxies for older adults. Although this is true, we again stress that researchers have used large sets of pre-existing public health survey data not intended for persona construction to create reasonable personas for European older adults [51]. Furthermore, we believe the BRFSS is more suitable to 
creating care-management personas than other potential health surveys which do not include behavior risk, chronic conditions and health literacy data, as we feel these factors are both highly relevant to representing the health condition and understanding of older adults for providing effective healthcare.

Although we provide reasonably consistent, novel, and potentially useful results, we agree that future work is needed to further validate and refine our care-management personas. We believe that the utilization of different sets of public data (e.g., the National Health Interview Survey, National Health and Nutrition Examination Survey, etc.) would be useful in accomplishing that verification of the robustness of our methods and resulting personas. Ideally, future works would also utilize project specific and tailored surveys to properly refine our caremanagement personas. We believe this empirical grounding is necessary, as without it, personas are merely stereotypes which may or may not represent older adults. Our work is thus grounded in persona research which constructs personas based on empirical evidence. Researchers should be aware that the design of the survey questions, as well as the content covered, will affect the resulting persona skeletons from these methods.

Utilization of Our Methods and Personas. Our quantitative methodology for creating persona skeletons is this work's primary contribution; our method is likely applicable to any imbalanced dataset, but we will discuss primarily our study context and results in this section. Our skeletons are quantitatively derived from real-world data, and thus offer a representative framework based on real individuals. However, the persona narratives created from these frameworks still required qualitative interpretation and creative activities on the part of the narrative writer. Therefore, our method allows for designers to have a quantitative framework for their qualitative narratives. We note our example case created highly generalized personas for U.S. older adults, as they represent all of the respondents from the nation; we therefore may not capture smaller scale trends (e.g., region-specific, condition-specific, etc). If researchers are interested in designing technologies to target specific subsets of older adults (e.g., regions, races, genders, etc.), repeating our methods using more targeted initial datasets would be the best method for ensuring accurate representation of smaller trends. Designers may also attempt to flesh out our persona skeletons in their early design stages by adding targeted data. Notably, our persona narratives were constructed using the quantitatively derived frameworks in Tables 1 and 2; as a result, any data not in these tables would theoretically be replaceable, as they were deemed insignificant. When replacing features for persona narratives, consider the following rules: 1) keep coherent narratives (i.e., do not create contradictions or else the persona narratives become invalid), and 2) do not alter any feature which was deemed significant.

Future works. Future works could utilize our personas for care-management research which emphasizes empathetic design approaches. For such user-centered design projects, we recommend the utilization of our care management personas in conjunction with scenarios, another user centered design method, as scenarios can be written directly based upon our persona narratives $[8,46]$. In addition, although our personas are not our main contribution, we would like to see them compared and contrasted with, and thus validated against, personas constructed by other methods.

Limitations. Several limitations should also be considered before using empirical methods similar to ours. While utilizing K-means and iterative feature selection increased the objectivity of persona construction, human judgment, and thus subjectivity, was necessary for the construction of persona narratives. Bias may have been introduced at this stage. Also, $\mathrm{K}$-means requires a predefined cluster number, so our four clusters may not represent the ideal cluster number for this dataset. We provide the source code so that other researchers can adjust the number of cluster centers they deem appropriate for their projects. Furthermore, like other older adult personas constructed from health survey data [51], our personas do not contain technology usage pattern information necessary for the design of healthcare technologies for older adults; our care-management personas could be used as the bulk of a persona for such projects, but would need to be updated with that critical technology use data. We must also clarify that personas are fictional representations that do not represent any one individual or replace human interaction in the design process; they are merely tools which can help designers better empathize and plan for human interactions to ensure that attention is drawn to relevant areas of interest and that needs are met. Finally we note that although our method is suitable for creating persona skeletons from large imbalanced data sets, our personas themselves may not be suitable for representing older adults from, for example, smaller regional trends. More targeted personas may be needed to represent smaller regions.

\section{CONCLUSION}

To briefly summarize this work, we developed a quantitative method to analyze a large public data resource and synthesize our results into a small collection of personas. Our 6-step persona creation methodology novelly utilizes a two-level approach to account for dataset discrepancies. This work offers clear documentation of how we constructed care-management persona skeletons from discrepant datasets from the BRFSS for this case study. We were motivated by the need and demand to increase empathy in care-management for older adults in the USA, as well as the need for quantitative persona construction methods. In addition to our method, which is applicable to other imbalanced datasets, we offer discussion regarding the potential use of our resulting example personas.

\section{ACKNOWLEDGEMENT}

The authors would like to acknowledge the National Science Foundation grant \#1502176 for funding this work.

\section{REFERENCES}

[1] 2019. Behavior Risk Factor Surveillance System. https: //www.cdc.gov/brfss/annual_data/annual_2016.html. (2019). Accessed: 2019-01-18.

[2] 2019. National Health and Nutrition Examination Survey. https://wwwn.cdc.gov/nchs/nhanes/ continuousnhanes/default . aspx?BeginYear=2016. (2019). Accessed: 2019-01-18. 
[3] 2019. National Health Interview Survey. https: //www.cdc.gov/nchs/nhis/nhis_2016_data_release.htm. (2019). Accessed: 2019-01-18.

[4] Muna S Al-Razgan, Hend S Al-Khalifa, and Mona D Al-Shahrani. 2014. Heuristics for evaluating the usability of mobile launchers for elderly people. In International Conference of Design, User Experience, and Usability. Springer, 415-424.

[5] Alissa Nicole Antle. 2006. Child-personas: fact or fiction?. In Proceedings of the 6th conference on Designing Interactive systems. ACM, 22-30.

[6] Christopher Brooks and Jim Greer. 2014. Explaining predictive models to learning specialists using personas. In Proceedings of the Fourth International Conference on Learning Analytics And Knowledge. ACM, 26-30.

[7] Frederick P Brooks Jr. 2010. The design of design: Essays from a computer scientist. Pearson Education.

[8] John M Carroll. 1995. Scenario-based design: envisioning work and technology in system development. (1995).

[9] Roberto Casas, Rubén Blasco Marín, Alexia Robinet, Armando Roy Delgado, Armando Roy Yarza, John Mcginn, Richard Picking, and Vic Grout. 2008. User modelling in ambient intelligence for elderly and disabled people. In International Conference on Computers for Handicapped Persons. Springer, 114-122.

[10] Yen-ning Chang, Youn-kyung Lim, and Erik Stolterman. 2008. Personas: from theory to practices. In Proceedings of the 5th Nordic conference on Human-computer interaction: building bridges. ACM, 439-442.

[11] Christopher N Chapman and Russell P Milham. 2006. The personas' new clothes: methodological and practical arguments against a popular method. In Proceedings of the human factors and ergonomics society annual meeting, Vol. 50. SAGE Publications Sage CA: Los Angeles, CA, 634-636.

[12] Dana E Chisnell, Janice C Ginny Redish, and AMY Lee. 2006. New heuristics for understanding older adults as web users. Technical Communication 53, 1 (2006), 39-59.

[13] A Cooper. 2000. The Inmates Are Running the Asylum, 1999. Indianapolis, Indiana: SAMS, A Division of Macmillan Computer Publishing (2000).

[14] Rikke Dam and Teo Siang. 2018. Personas - Why and How You Should Use Them.

https://www.interaction-design.org/literature/ article/personas-why-and-how-you-should-use-them. (2018). Accessed: 2018-04-15.

[15] John Partomo Djajadiningrat, William W Gaver, and JW Fres. 2000. Interaction relabelling and extreme characters: methods for exploring aesthetic interactions. In Proceedings of the 3rd conference on Designing interactive systems: processes, practices, methods, and techniques. ACM, 66-71.
[16] Janna Lynn Dupree, Richard Devries, Daniel M Berry, and Edward Lank. 2016. Privacy personas: Clustering users via attitudes and behaviors toward security practices. In Proceedings of the 2016 CHI Conference on Human Factors in Computing Systems. ACM, 5228-5239.

[17] Shamal Faily and Ivan Flechais. 2011. Persona cases: a technique for grounding personas. In Proceedings of the SIGCHI Conference on Human Factors in Computing Systems. ACM, 2267-2270.

[18] Centers for Disease Control, Prevention, and others. 2016. Behavioral risk factor surveillance system survey data. Atlanta, GA: US Department of Health and Human Services, Centers for Disease Control and Prevention. (2016).

[19] Denae Ford, Thomas Zimmermann, Christian Bird, and Nachiappan Nagappan. 2017. Characterizing Software Engineering Work with Personas Based on Knowledge Worker Actions. In 2017 ACM/IEEE International Symposium on Empirical Software Engineering and Measurement (ESEM). IEEE, 394-403.

[20] Jason Fried. 2007. Ask 37signals: Personas? https: //signalvnoise. com/posts/690-ask-37signals-personas. (2007). Accessed: 2018-04-15.

[21] Jerome Friedman, Trevor Hastie, and Robert Tibshirani. 2001. The elements of statistical learning. Vol. 1. Springer series in statistics New York, 506-507.

[22] Kim Goodwin. 2002. Getting from research to personas: harnessing the power of data. https://www. cooper.com/ journal/2002/11/getting_from_research_to_perso. (2002). Accessed: 2018-04-15.

[23] Jonathan Grudin and John Pruitt. 2002. Personas, participatory design and product development: An infrastructure for engagement. In PDC. 144-152.

[24] Hang Guo and Khasfariyati Binte Razikin. 2015. Anthropological User Research: A Data-Driven Approach to Personas Development. In Proceedings of the Annual Meeting of the Australian Special Interest Group for Computer Human Interaction. ACM, 417-421.

[25] Melissa Harper and Patricia Cole. 2012. Member checking: can benefits be gained similar to group therapy? The Qualitative Report 17, 2 (2012), 510-517.

[26] Dallas M High and Mary M Doole. 1995. Ethical and legal issues in conducting research involving elderly subjects. Behavioral sciences \& the law 13, 3 (1995), 319-335.

[27] Charles G Hill, Maren Haag, Alannah Oleson, Chris Mendez, Nicola Marsden, Anita Sarma, and Margaret Burnett. 2017. Gender-Inclusiveness Personas vs. Stereotyping: Can We Have it Both Ways?. In Proceedings of the 2017 CHI Conference on Human Factors in Computing Systems. ACM, 6658-6671. 
[28] Lawrence Hubert and Phipps Arabie. 1985. Comparing partitions. Journal of classification 2, 1 (1985), 193-218.

[29] Marc Johannes, Jan C Brase, Holger Fröhlich, Stephan Gade, Mathias Gehrmann, Maria Fälth, Holger Sültmann, and Tim Beißbarth. 2010. Integration of pathway knowledge into a reweighted recursive feature elimination approach for risk stratification of cancer patients. Bioinformatics 26, 17 (2010), 2136-2144.

[30] David A Leon. 2011. Trends in European life expectancy: a salutary view. (2011).

[31] Cynthia LeRouge, Jiao Ma, Sweta Sneha, and Kristin Tolle. 2013. User profiles and personas in the design and development of consumer health technologies. International journal of medical informatics 82, 11 (2013), e251-e268.

[32] Carol Levine, Ruth Faden, Christine Grady, Dale Hammerschmidt, Lisa Eckenwiler, and Jeremy Sugarman. 2004. The limitations of "vulnerability" as a protection for human research participants. The American Journal of Bioethics 4, 3 (2004), 44-49.

[33] Russell Marshall, Sharon Cook, Val Mitchell, Steve Summerskill, Victoria Haines, Martin Maguire, Ruth Sims, Diane Gyi, and Keith Case. 2015. Design and evaluation: End users, user datasets and personas. Applied ergonomics 46 (2015), 311-317.

[34] Andrey A Masiero, Mayara G Leite, Lucia Vilela Leite Filgueiras, and Plinio Thomaz Aquino Jr. 2011. Multidirectional knowledge extraction process for creating behavioral personas. In Proceedings of the 10th Brazilian Symposium on Human Factors in Computing Systems and the 5th Latin American Conference on Human-Computer Interaction. Brazilian Computer Society, 91-99.

[35] Jennifer Jen McGinn and Nalini Kotamraju. 2008. Data-driven persona development. In Proceedings of the SIGCHI Conference on Human Factors in Computing Systems. ACM, 1521-1524.

[36] Tomasz Miaskiewicz and Kenneth A Kozar. 2011. Personas and user-centered design: How can personas benefit product design processes? Design Studies 32, 5 (2011), 417-430.

[37] Tomasz Miaskiewicz, Tamara Sumner, and Kenneth A Kozar. 2008. A latent semantic analysis methodology for the identification and creation of personas. In Proceedings of the SIGCHI Conference on Human Factors in Computing Systems. ACM, 1501-1510.

[38] Steve Mulder and Ziv Yaar. 2006. The user is always right: A practical guide to creating and using personas for the web. New Riders.

[39] Don Norman. 2004. Ad-hoc personas \& empathetic focus. Jnd. org (2004).

[40] Marija J Norusis and Sppss Inc. 2010. PASW statistics 18: Statistical procedures companion. Prentice-Hall.

[41] Francisco Nunes, Paula Alexandra Silva, and Filipe Abrantes. 2010. Human-computer interaction and the older adult: an example using user research and personas. In Proceedings of the 3rd international conference on PErvasive technologies related to assistive environments. ACM, 49.

[42] Rich Picking, Alexia Robinet, Vic Grout, John McGinn, Armando Roy, Simon Ellis, and Denise Oram. 2009. A case study using a methodological approach to developing user interfaces for elderly and disabled people. Comput. J. 53, 6 (2009), 842-859.

[43] John Pruitt and Tamara Adlin. 2010. The persona lifecycle: keeping people in mind throughout product design. Elsevier.

[44] John Pruitt and Jonathan Grudin. 2003. Personas: practice and theory. In Proceedings of the 2003 conference on Designing for user experiences. ACM, $1-15$.

[45] Paul Ramcharan and John R Cutcliffe. 2001. Judging the ethics of qualitative research: considering the 'ethics as process' model. Health \& social care in the community 9, 6 (2001), 358-366.

[46] Mary Beth Rosson and John M Carroll. 2009. Scenario based design. Human-computer interaction. boca raton, FL (2009), 145-162.

[47] Peter J Rousseeuw. 1987. Silhouettes: a graphical aid to the interpretation and validation of cluster analysis. Journal of computational and applied mathematics 20 (1987), 53-65.

[48] Kishan Salian. 2018. UX Persona - The Introduction. https://medium. com/swlh/ ux-persona-the-introduction-3aba2e5237df. (2018). Accessed: 2018-04-15.

[49] Susan A Walsh. 2009. Conducting research with the elderly: Ethical concerns for a vulnerable population. Southern Online Journal of Nursing Research 9, 4 (2009), 1-13.

[50] Ian H Witten, Eibe Frank, Mark A Hall, and Christopher J Pal. 2016. Data Mining: Practical machine learning tools and techniques. Morgan Kaufmann.

[51] Bernhard Wöckl, Ulcay Yildizoglu, Isabella Buber, Belinda Aparicio Diaz, Ernst Kruijff, and Manfred Tscheligi. 2012. Basic senior personas: a representative design tool covering the spectrum of European older adults. In Proceedings of the 14th international ACM SIGACCESS conference on Computers and accessibility. ACM, 25-32.

[52] Svante Wold, Kim Esbensen, and Paul Geladi. 1987. Principal component analysis. Chemometrics and intelligent laboratory systems 2, 1-3 (1987), 37-52.

[53] Jennifer L Wolff, Barbara Starfield, and Gerard Anderson. 2002. Prevalence, expenditures, and complications of multiple chronic conditions in the elderly. Archives of internal medicine 162, 20 (2002), 2269-2276. 